\title{
Sensors and instrumentation for monitoring and control of multi-phase separation ${ }^{*}$
}

\author{
by Wuqiang Yang \\ School of Electrical and Electronic Engineering, The University of Manchester
}

\section{Introduction}

Multi-phase separation, in particular crude oil separation, is an important process in the oil industry. Usually, crude oil from oil wells is pumped to large tanks (typically 2 meters in diameter and 5-10 meters in length) to separate gas, oil, water and other materials, such as sand, aiming to extract oil. Most crude oil separators rely on gravity separation and the separation process takes a long time. To optimise the use of separators, it is important to understand the internal behaviour of the separators, i.e. it is important to monitor the separation process and to measure the multi-interfaces between different materials, such as gas-foam, foam-oil, oil-emulsion, emulsion-water and water-sand (see Fig. 1).

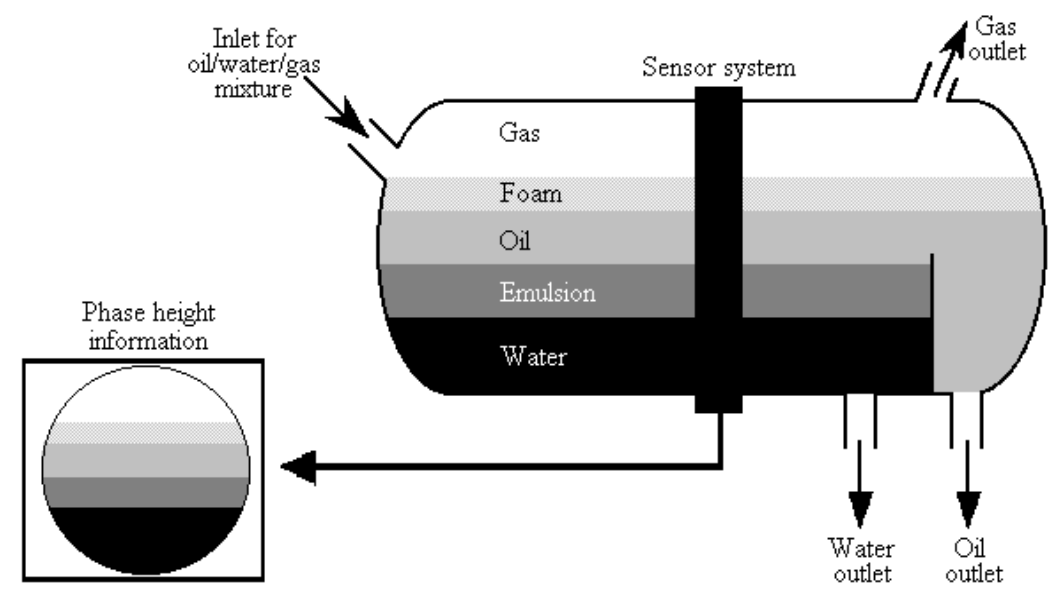

Fig. 1 Typical crude oil separator (Isaksen et al. 1994)

Multi-interface measurement is particularly important to the offshore oil companies because crude oil from undersea contains significant amount of water. Ideally, down-hole on-line separation of water can be implemented. To be more realistic, water may be separated from a crude oil stream on offshore oil platforms rather than transported to the onshore facilities because the transportation process is expensive. Because the space on offshore oil platforms is limited and expensive, effective monitoring and control of the crude oil separation process on offshore oil platforms will result in huge saving to the oil companies. Therefore, it is necessary to have reliable instruments to monitor the separation process and to measure multi-interfaces for the benefit of oil companies. Accurate measurement of the interface levels and fluid components will also benefit environment by reducing pollution.

While the conventional gravity-based crude oil separators are operated off-line, some companies have developed new types of on-line crude oil separators. For example, Merpro has developed a compact three-phase separator TORE SEP ${ }^{\circledR}$, which can be used to separate oil, water and gas three components on-line (Merpro, 2005). The separator is based on the cyclone principle with a tangential acceleration of up to $50 \mathrm{~g}$, providing rapid and effective oil-water separation. As shown in Fig. 2, the separated oil flows through the central discharge pipe, gas discharges from the top and water discharges tangentially from the separator. It is small and lightweight and hence attractive for offshore installation. While it is equally important to understand the internal behaviour of this type of separation facility, the following will mainly discuss measurement of multi-interfaces in gravity-based separators.

* This paper was presented at Multiphase Separation and Multiphase Pumping Technologies Conference, 1-2 September 2005, Aberdeen, UK 


\section{Conventional sensors and instrumentation}

While many different types of sensors or transducers for single interface level measurement are available on market, such as capacitance, magnetic and ultrasonic, few types of sensors and instrumentation have been developed to monitor the operation of crude oil separators and to measure multi-interface levels in crude oil separators (Bukhari and Yang 2005).

$\gamma$-ray systems

Among the multi-interface level measurement systems ( $\gamma$-ray, ultrasonic and capacitance) have been developed, $\gamma$-ray systems, e.g. the Tracerco Profiler (see Fig. 2) from Tracerco, the former ICI Synetix, may be regarded as the most reliable for measuring multi-interfaces in crude oil separators (Darwood et al. 2003, Tracerco 2005, Lees 2005, Hjertaker et al. 2001). It is well known that this type of system is based on measuring attenuation of $\gamma$ radiation to detect the density profile in a separator. The Tracerco Profiler uses two (or three) rods vertically installed inside of a separator, a source rod consisting of an array of low energy $\gamma$ radiation sources collimated to produce narrow beams as $\gamma$ emitters and a detection rod holding an array of corresponding $\gamma$ detectors (see Fig. 3). Because of different densities of oil, water, emulsion, foam and sand, the radiation signals are attenuated differently and hence the multiinterfaces can be measured accordingly.

While this type of systems has proven to be accurate and reliable, it has some drawbacks:

- It uses radiation sources, which causes safety concerns and potentially health problems,

- It is expensive to purchase and expensive to maintain,

- In some countries, e.g. in EU countries, radiation-based instruments may not be allowed to use in open space.

While the Tracerco Profiler is working in the transmission mode, some other systems have been developed also based on $\gamma$ radiation but in scattering mode, such as that investigated at University of Bergen (Holstad 2003).

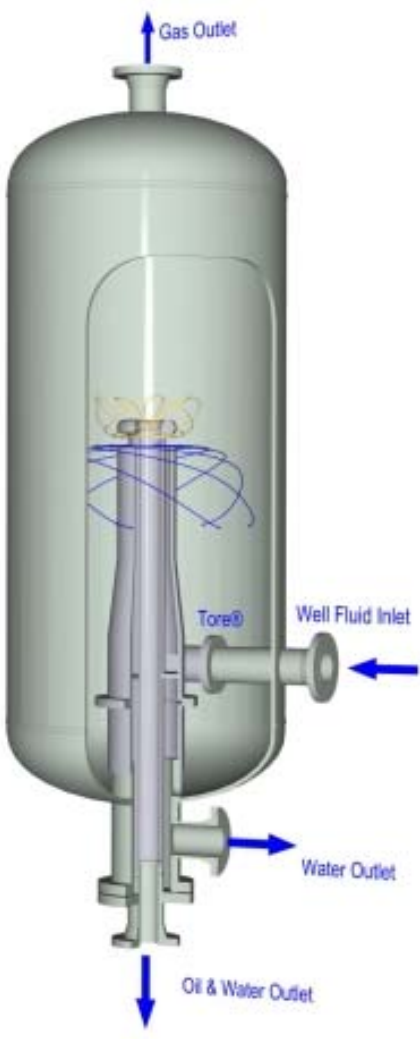

Fig. 2 Three-phase separator (Merpro 2005)

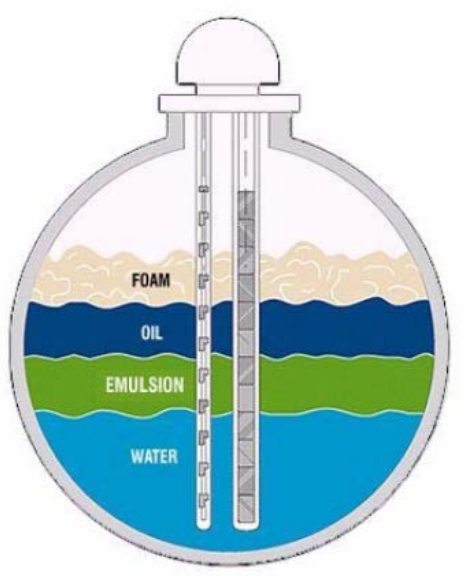

Fig. 3 -ray based Tracerco Profiler (Tracerco 2005)

\section{Ultrasonic systems}

Ultrasonic transducers have been used for single-level measurement for many years. They are good at determining a single interface between gas-liquid or gas-solids. It is common to use an ultrasonic transducer in the reflection mode. The distance between the transducer and the interface is determined by the flight-of-time. Some companies, such as Christian Michelsen Research (CMR) in Norway, have developed ultrasonic systems for multi-interface measurement, in particular for the use in crude oil 
separators. Fig. 4 shows the CMR ultrasonic interface level detector (CMR 2005). The system consists of a clamp-on ultrasonic sensor, sensing electronics for hazardous zone, a zone interface with ex-barriers, power supply and a processing unit with a monitor. The clamp-on ultrasonic sensor is mounted with direct contact to the outside wall at the bottom of a separator, transmitting and receiving ultrasonic pulses along the vertical axis. The operation is based on pulse-echo and interface levels are estimated by flightof-time of echoes from the interfaces with consideration of the speed of sound in the propagation media.

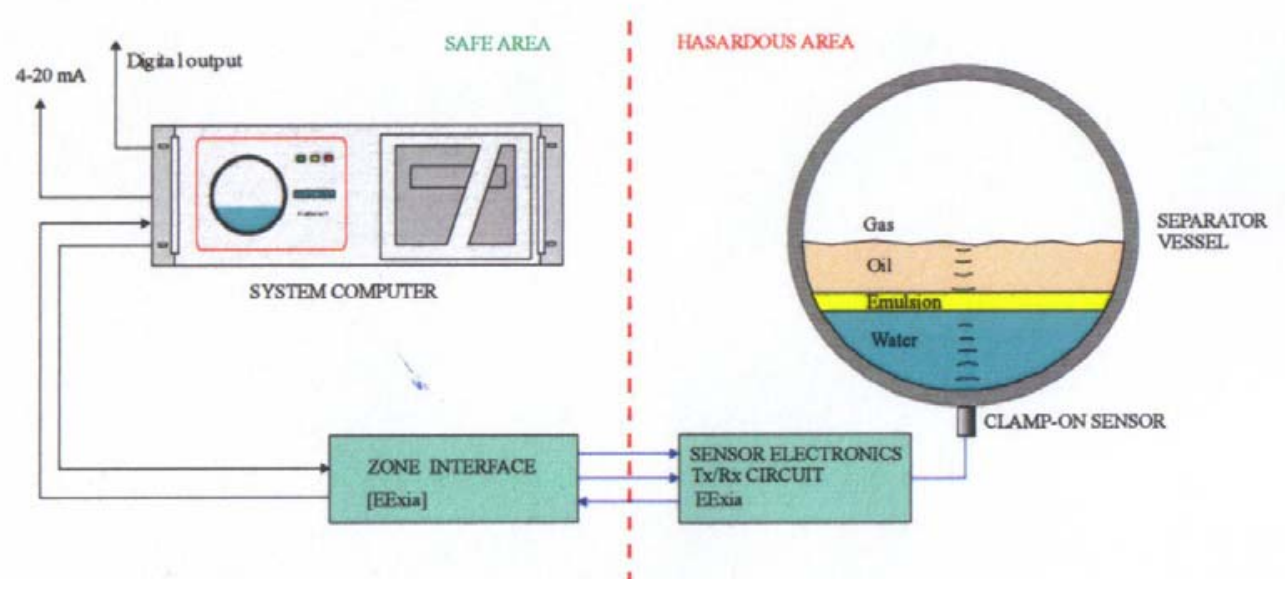

Fig. 4 Ultrasonic interface level detector (CMR 2005)

There are some problems associated with ultrasonic transducers to be used for multi-interface level measurement (Beck et al. 1994, Hjertaker et al. 2001):

- The ultrasonic signal is attenuated by the first interface significantly and hence the echoes from the second and further interfaces would be too weak to detect.

- $\quad$ To deal with attenuation of signal due to the vessel wall, sufficient signal power needs to be applied. However, there is a limit to the energy applied by intrinsic safety. If the ultrasonic transducer is instead mounted inside of a separator, it becomes intrusive and again it causes intrinsic safety concerns.

- $\quad$ Foam may completely absorb ultrasonic energy and thus a layer of foam will cause false reading.

\section{Capacitance systems}

Most capacitance transducers adopt the single rod structure with a single capacitance electrode while the vessel wall is used as another electrode. This type of transducer is designed to measure a single interface level. A typical example is the E+H Rod probes 11500 from Endress + Hauser (2005), which is designed for use in explosion-hazardous area Zone 0 . The ceramic rod probe is fully insulated for hightemperature and high-pressure (HTHP) applications. It can measure a continuous level but is limited to detection of liquids. Even with a single interface, this type of transducer is not accurate because capacitance is a function of temperature. That means that change in temperature can affect capacitance measurement significantly and gives inaccurate location of an even single interface between two different materials (Lees 2005).

The first capacitance-based instrumentation for multi-interface level measurement was developed by Shell (1984). The sensor probe consists of two parallel plates facing each other, one with a single electrode for excitation and the other with segmented electrodes for detection. One of the problems with this design is that the slot between the two plates can be easily blocked by sticky oil. The system was used for measurement of a single interface in petrol storage tanks (Enraf-Nonius 1988).

Shi et al. (1991) developed the first single capacitance rod with a multi-electrode array. By measuring fringe capacitance of each adjacent electrode pair, different materials, such as gas, oil and water, may be identified and the interfaces between different materials may be located. The resolution is limited to the height of one electrode. 
In 1992, a project "Interface measurement in three-phase separators" as a part of "Design and instrumentation of primary separation systems - Phase I” was funded by Science and Engineering Research Council (SERC) and Marinetech North West (UK), a consortium including several universities and offshore oil companies. Research into multi-interface level measurement using segmented capacitance sensors was carried out in the Department of Electrical Engineering and Electronics at UMIST until 1994. During this time period, a parallel-plate multi-electrode capacitance sensor together with a capacitance measuring circuit and a data acquisition system was developed.

As shown in Fig. 5, the sensor consists of a single detection electrode and 32 excitation electrodes (Yang et al. 1994, Asran 1998, Akhneifer 1999, Yang 2001). Capacitance is measured between each of the excitation electrodes and the detection electrode. An advantage of this arrangement is that the multiplexer between the excitation electrodes and the excitation source does not cause coupling problems. If a multiplexer is placed between multiple detection electrodes and a common measuring circuit, the coupling capacitance of the multiplexer would cause problems because the preliminary signal is small and the coupling capacitance would be in the same order as the unknown capacitance to be measured. Due to different permittivity values for gas $\left(\varepsilon_{\mathrm{r}}=1.0\right)$, oil $\left(\varepsilon_{\mathrm{r}}\right.$ between 2.1 and 2.6, depending on type of oil) and water $\left(\varepsilon_{\mathrm{r}}=80\right)$, the measured capacitance can reflect what material is in between the detection electrode and a specific excitation electrode.

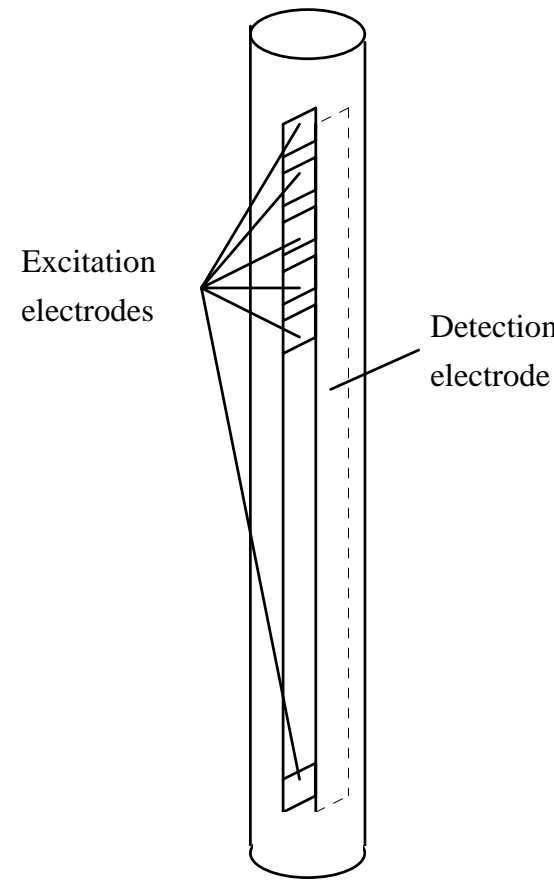

(a) $3 D$ illustration

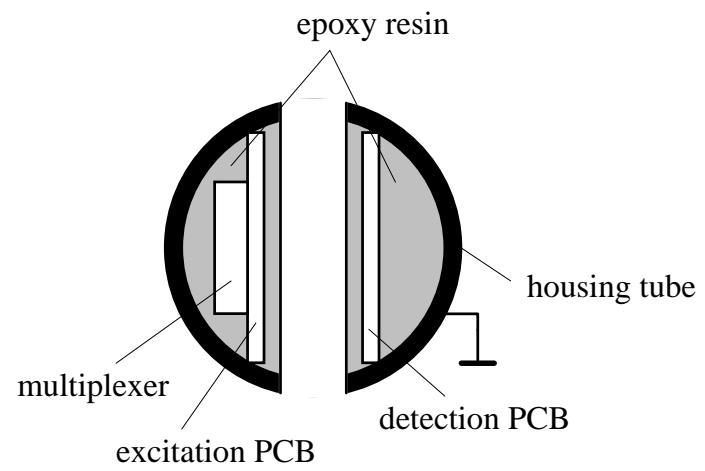

(b) Cross-section view

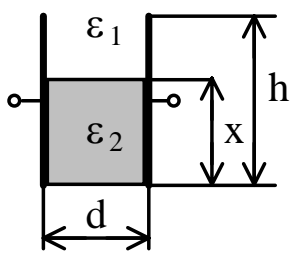

(c) Capacitance between the detection electrode and an excitation electrode

Fig. 5 Segmented capacitance sensor developed at UMIST

For this designed sensor, the capacitance to be measured is small, a few Pico Farads (pF). Therefore, a high-sensitivity, low-noise, stray-immune capacitance measuring circuit is needed. For this project, an AC-based circuit was used, which employs high-frequency sine-wave excitation and phase-sensitive demodulation. The sensor and the system were tested in laboratory successfully to locate the interfaces of air-oil and oil-water (Yang 2001).

The sensor was designed to fit to 2" installation hole, to be resistant to corrosion, heat and pressure and to be intrinsically safe. Because of multi-electrodes facing different materials, the measured data can be used to calibrate each other and an interface level may be estimated more accurately by interpolation. For example, if an electrode pair is entirely in air $\left(\varepsilon_{1}\right)$ and another electrode pair is entirely in oil $\left(\varepsilon_{2}\right)$, then the capacitance of the third electrode pair, where an interface level locates as shown in Fig. 5 (c), can be calculated by 


$$
C=\frac{\varepsilon_{0} w h}{d}\left[\varepsilon_{1}+\left(\varepsilon_{2}-\varepsilon_{1}\right) \frac{x}{h}\right]
$$

where, $\mathrm{C}$ is the measured capacitance of the third electrode pair $(\mathrm{pF}), \varepsilon_{\mathrm{o}}$ is the permittivity of free space $(8.85 \mathrm{pF} / \mathrm{m}), \varepsilon_{1}$ and $\varepsilon_{2}$ are dielectric constants of two different materials (air and oil in this case), $d$ is the distance between two electrodes (m), $w$ and $h$ are the width and height of electrode respectively (m), and $x$ is the interface level above the bottom of the third electrode pair (m).

From the above equation, the level within the third electrode can be easily derived. Obviously, the parallel-plate capacitance sensors can give better resolution than the single capacitance plate sensor. Another feature of this sensor is that it can be used to detect foam layer by Fourier analysis of fluctuation in capacitance due to the nature of instability of foam (Yang et al. 1994).

The major problem with this multi-electrode capacitance sensor is that if an electrode is merged in highly conductive liquid, such as saline water, high conductivity would make the electrodes short-circuit (Hjertaker et al. 2001). Considering that in most cases crude oil contains saline water, this sensor is unable to work with a real crude oil separator.

Several institutions have followed this design and the work by Shi et al. to further develop multielectrode capacitance level systems, e.g. Tianjin University in China (Wang et al. 1996, Wang and Yin 1997), Agricultural University of Norway (Schüller et al. 2001) and the Department of Chemical Engineering at UMIST (now the School of Chemical Engineering and Analytical Science at the University of Manchester) (Jaworski et al. 1999, Jaworski and Dyakowski 2005). Some improvements have been made since then, which may be summarised as follows.

At Tianjin University, a comb-structure as shown in Fig. 6 was adopted, aiming to achieve higher sensitivity. Basically, this design is similar to that developed by Shi et al. in 1991. It was reported that this level system was tested in the Zhong Yuan Oilfield successfully.

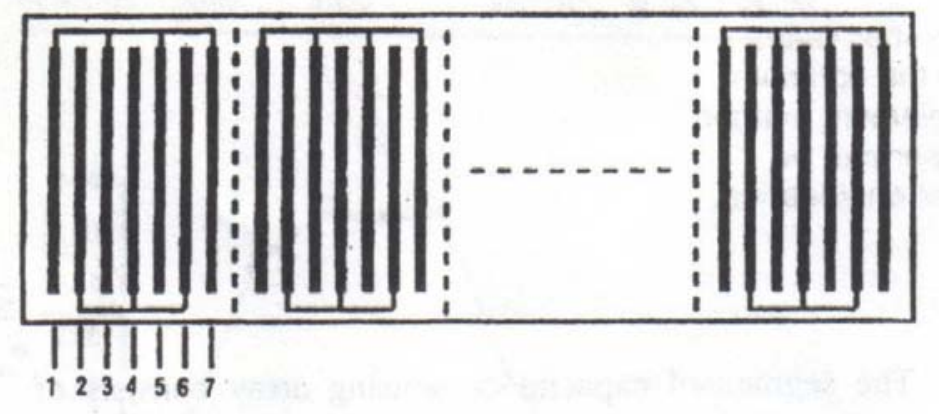

Fig. 6 Comb-structure segmented capacitance sensor developed at Tianjin University

At Agricultural University of Norway, a single electrode array was developed, which is also similar to the design reported by Shi et al. (1991). The main difference is that the Agricultural University's sensing circuits were integrated onto the sensor. It was reported that this level system was tested in Norsk Hydro successfully.

Following the work done in the Department of Electrical Engineering and Electronics at UMIST, further work was done in the Department of Chemical Engineering (Jaworski et al. 1999a, Jaworski et al. 1999b). A new development is that a capacitance-ultrasonic dual-modality probe with thick-film electrodes was developed, aiming to deal with the fouling problems (Dyakowski et al. 2005, Meng et al. 2005). In this design, ultrasonic devices are used to vibrate the capacitance electrodes to get rid of fouling, not function as transducers. It was reported that the sensor was tested at the UMIST Pilot Plant, the BP Exploration Wytch Farm and the National Engineering Lab (NEL). However, making a thin-film electrode sensor would be quite expensive (Jaworski and Dyakowski 2005). 
In principle, conductive water can cause shorting-circuit of capacitance electrodes. This fundamental limitation makes it almost impossible to use capacitance sensors only for measurement of multi-interface levels in crude oil separators (Hjertaker et al. 2001).

\section{Inductive systems}

In addition to $\gamma$-ray, ultrasonic and capacitance, other types of sensors have also been investigated as alternative or complementary sensing modalities. At UMIST, research into multi-interface measurement, in particular with saline water, using Hall-effect sensors and inductance coils was carried out (Syed 2004, Akhneifer 1999). It has been confirmed experimentally that the interface between water and oil can be detected reliably by a Hall-effect sensor or an inductance coil, either with or without salt.

ABB developed an inductive level monitoring system for multi-interface level measurement. In this design, multiple inductive coils are mounted on a rod (see Fig. 7). The induced current depends on the conductivity of the surrounding medium, which causes a counter-acting flux. By measuring the change in inductance, the conductivity can be estimated. It was confirmed experimentally that water or watercontinuous emulsion can be detected using this system without fouling problems, because coverage of scale or wax does not affect the magnetic measurement. However, it does not provide information in the region of oil and gas.

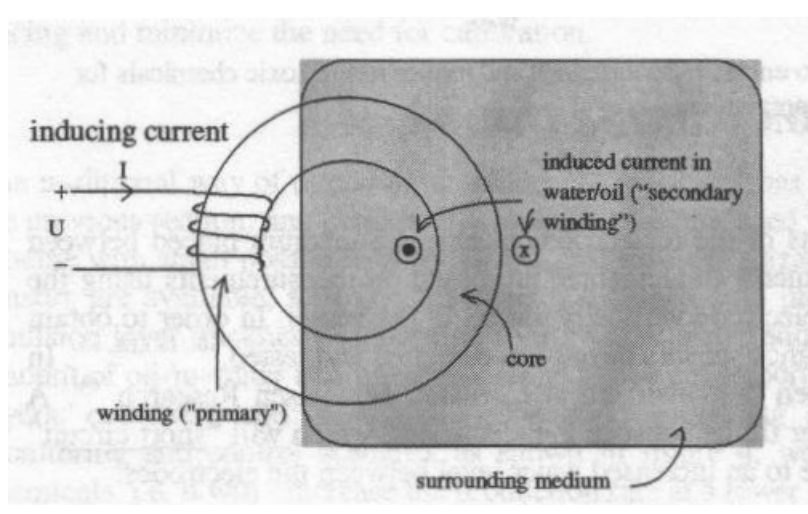

(a) Ferrite ring with an coil

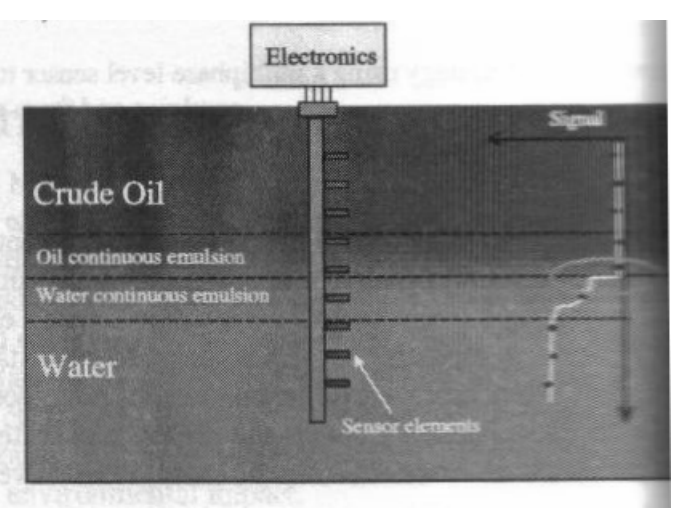

(b) Rod with multiple sensing elements

Fig. 7 ABB inductive level sensor (Hjertaker et al. 2001)

Table 1 gives a summary of different sensors for multi-interface measurement.

Table 1 Summary of different sensors

\begin{tabular}{|l|l|l|}
\hline Sensor & Main advantages & Main disadvantages \\
\hline$\gamma$-ray & Reliable and accurate & Radiation concerns, expensive \\
\hline Ultrasonic & $\begin{array}{l}\text { Non-intrusive and non-invasive, easy to } \\
\text { install }\end{array}$ & $\begin{array}{l}\text { Weak signal from second and further } \\
\text { interfaces }\end{array}$ \\
\hline $\begin{array}{l}\text { Single capacitance } \\
\text { plate }\end{array}$ & $\begin{array}{l}\text { Simple in structure } \\
\text { saline water }\end{array}$ \\
\hline $\begin{array}{l}\text { Parallel } \\
\text { capacitance plates }\end{array}$ & $\begin{array}{l}\text { More accurate than single capacitance } \\
\text { plate, foam detection }\end{array}$ & Fouling, difficulty with saline water \\
\hline $\begin{array}{l}\text { Capacitance- } \\
\text { ultrasonic }\end{array}$ & No-fouling & Expensive, difficulty with saline water \\
\hline ECT & $\begin{array}{l}\text { Non-intrusive and non-invasive, able to } \\
\text { measure all materials, including } \\
\text { emulsion }\end{array}$ & Expensive \\
\hline Inductive & $\begin{array}{l}\text { Detect water reliably, no-fouling } \\
\text { problems }\end{array}$ & Unable to detect oil and gas \\
\hline
\end{tabular}




\section{Tomography sensors and instrumentation}

As discussed above, all conventional sensors are invasive, which is not desirable in industry. Tomography provides a means of measuring internal behaviour of industrial processes non-intrusively and/or non-invasively. Among various sensing modalities, electrical tomography, which includes electrical capacitance tomography (ECT), electrical resistance tomography (ERT) and electro-magnetic tomography (EMT), is the first developed and the most mature. In particular, ECT has advantages of low cost, high imaging rate, both non-intrusive and non-invasive, no radiation, no moving parts and can withstand HTHP in harsh industrial environment.

Isaksen et al. (1994) made the first attempt to use ECT for measurement of multi-interfaces in an oil separator at CMR. As shown in Fig. 8 (a), eight capacitance electrodes were used to surround the oil separator of 1 meter in diameter. Fig. 8 (b) shows a cross-sectional view of an ECT sensor. Basically, capacitance is measured for each of all single-electrode combinations. In this case, there are 28 capacitance measurements available. From those measurements, the cross-sectional distribution of materials, i.e. a tomographic image, is reconstructed.

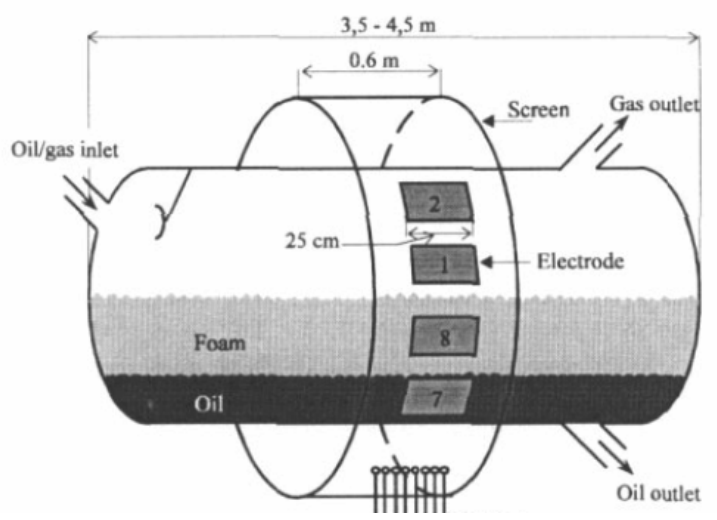

(a) Capacitance electrodes surrounding an oil separator (Isaksen 1994)

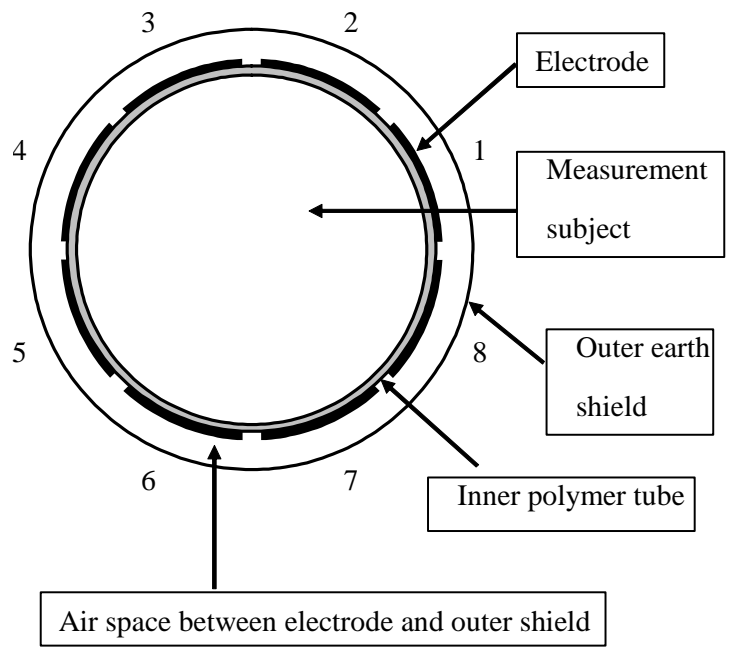

(b) Cross-sectional view of ECT sensor

Fig. 8 ECT sensor for measurement of multi-interfaces in oil separator

In this particular case, the following must be considered for image reconstruction:

- $\quad$ ECT is normally used to distinguish two dielectric materials of different permittivity. In an oil separator, however, there are more than two materials inside.

- The interfaces in an oil separator must be horizontal in a cross section as shown in Fig. 1. This is useful prior knowledge.

- Because the diameter of an oil separator is much larger than normal cases, between 2” and 4”, the fringe effect needs to be taken account.

- Imaging speed is not a concern because the process is slow.

In consideration of the above, Isaksen et al. (1992) developed an iterative algorithm called the explicit model based image reconstruction. In this algorithm, the distribution of gas, foam, oil, emulsion and water is assumed to be similar to that as shown in Fig. 1. When an initial guess image has been obtained by some algorithm, usually by linear back-projection (LBP), a set of capacitance can be calculated from the image and compared with a set of measured capacitance. If the initial image is exactly the same as the true distribution (this is unlikely the case), the set of calculated capacitance would be the same as the set of measured capacitance. If the initial image is different from the true distribution, there must be difference between the set of measured capacitance and the set of calculated capacitance. The difference is used to modify the interface levels so that the difference is reduced. In this way, the image is modified again and again until the difference between the set of measured capacitance and the set of calculated capacitance is sufficiently small. 
The main difference between this algorithm and other iterative algorithms is that with this algorithm the interfaces are assumed to be horizontal and only a few parameters, i.e. the interface levels, are modified during the iterative process. A weakness of this approach is that prior knowledge in material distribution must be known, which in many cases may not be available.

At the University of Manchester, a different approach has been used. To image air, oil and water, an ECT sensor is calibrated by air and oil first, and then it is calibrated by air and water to generate two references. When a set of capacitance has been acquired from an ECT sensor surrounding an oil separator, one image is generated using the air-oil calibration data to reflect the contrast between air as one component and oil and water as another component. A second image is then generated using the airwater calibration data to reflect the contrast between air and oil as one component and water as another component. The next step is to combine the two images together to illustrate three-component distribution as shown in Fig. 9.

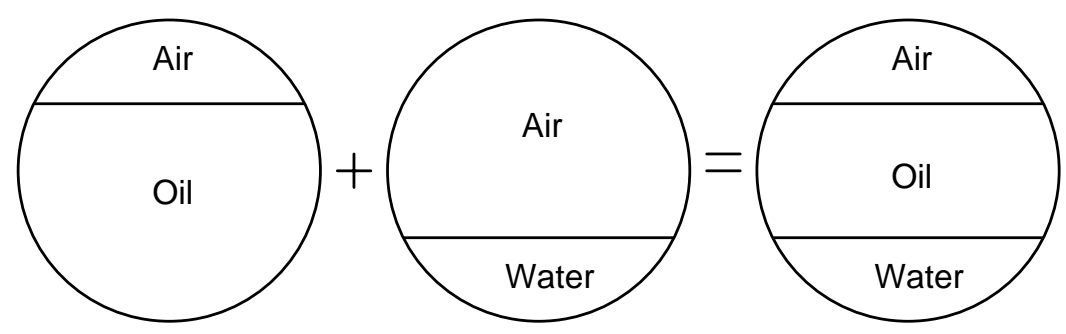

Fig. 9 Combine two images to show three-component distribution

The interface levels may also be located by knowledge-based analysis. In this case, many sets of capacitance data for different material distributions are saved as references. Once a set of capacitance data is obtained, a reference profile nearest to the measured capacitance profile is picked up to identify the interfaces.

In principle, an ECT-based sensor can give not only the interface information, but also the composition of emulsion, i.e. the percentages of water and oil in emulsion. This would help operators know more detailed information during the separation process.

\section{Control of oil separator}

Once the interfaces can be monitored, a natural development is to use the information for controlling the operation of oil separators. At the University of Manchester a test rig for investigation of oil separator control has been built. Fig. 10 shows the test rig, which consists of the following units:

- A separator of 4" in diameter mounted horizontally with 8 capacitance electrode surrounding it

- A mixer of 4 " in diameter mounted vertically with 8 capacitance electrodes surrounding it

- Two storage tanks, one for water and the other for oil

- An HP 4192A impedance analyser

- A multiplexer box with reed relay switches

- $\quad$ Pumps, valves and driving electronics.

Fig. 11 shows an impedance analyser based tomography system. Capacitance data are sampled from two ECT sensors, surrounding the separator and the mixer, to the impedance analyser via the multiplexer box, and then sent to a host PC. The PC communicates with the impedance analyser via IEEE488 and controls the multiplexer box via RS232 (Chondronasios et al. 2001). 


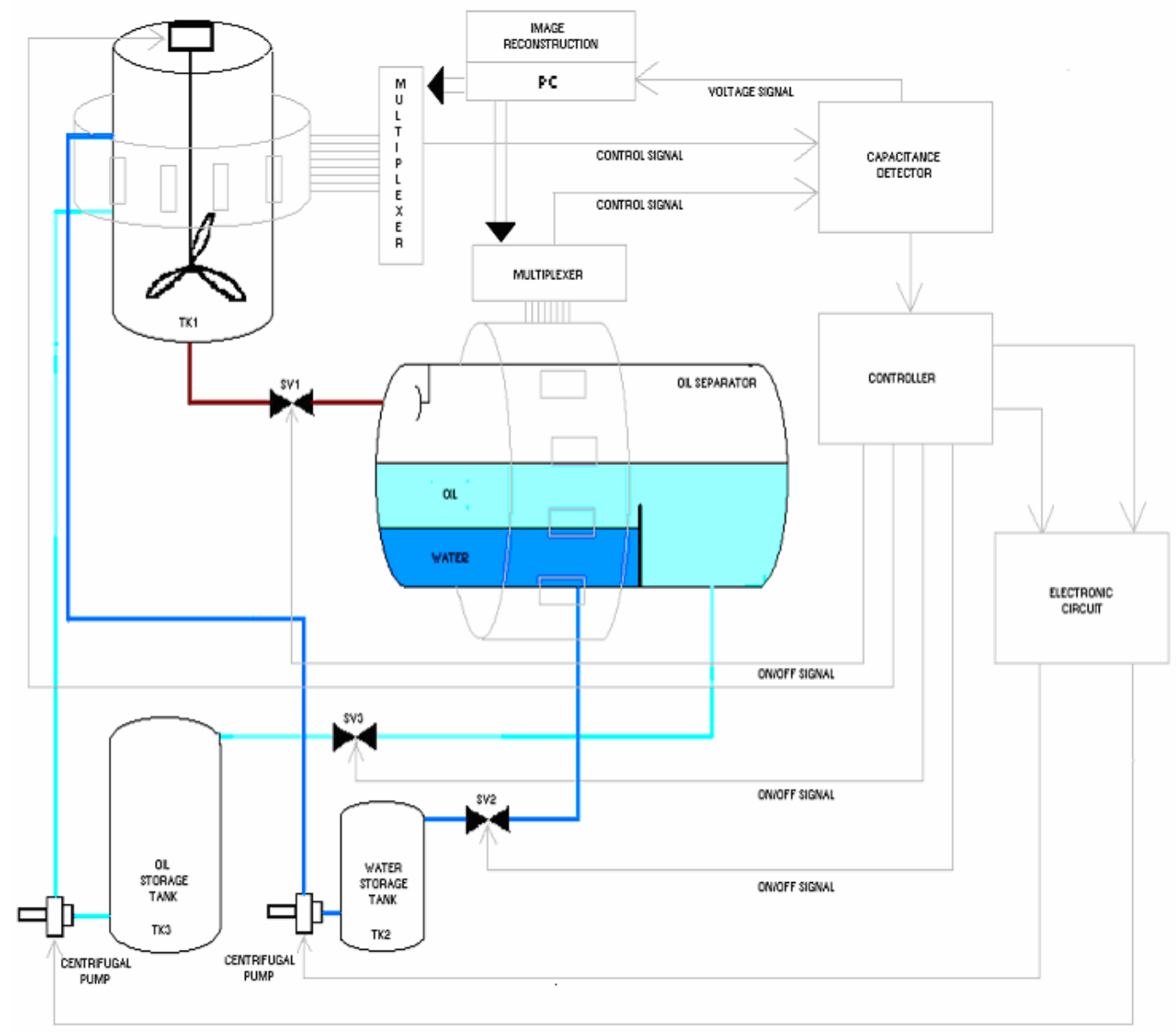

(a) Block diagram

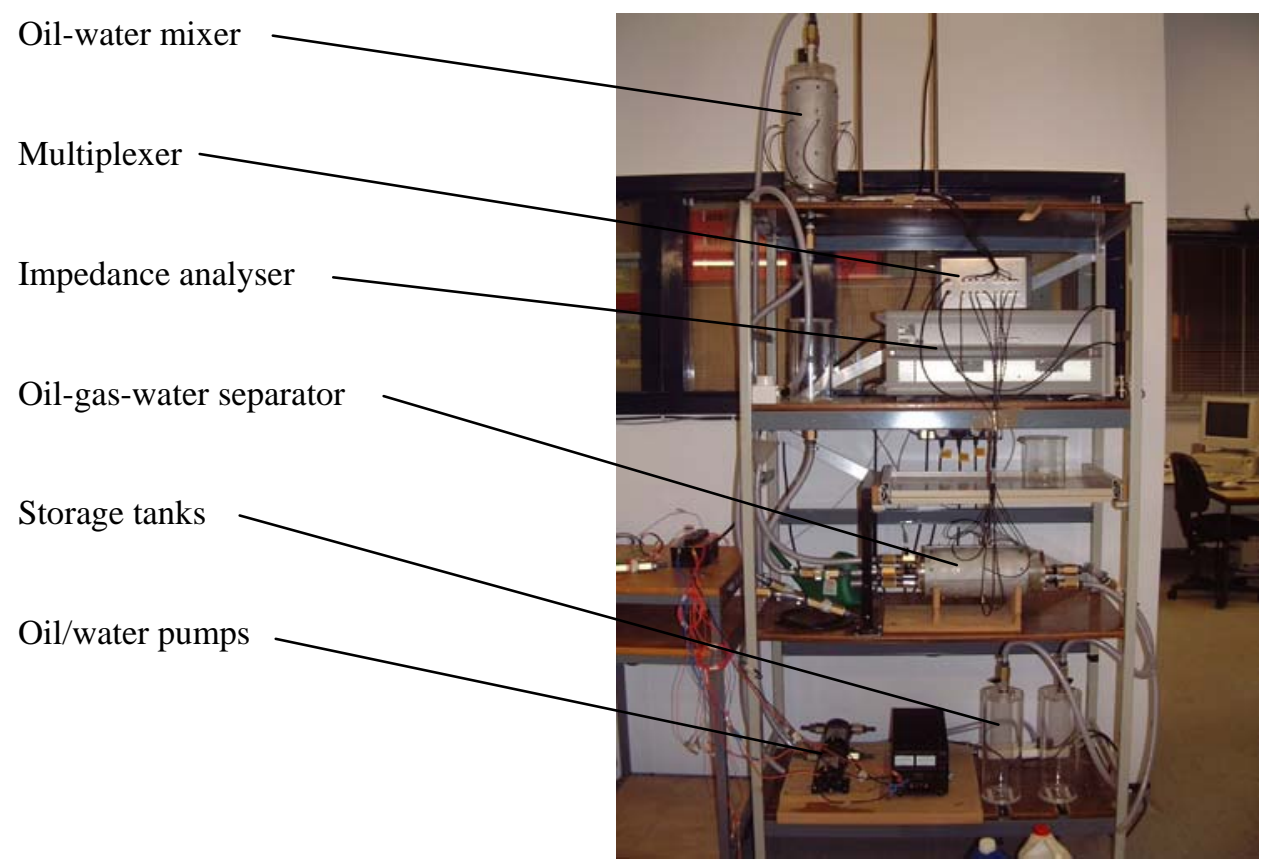

(b) Photo

Fig. 10 Test rig for oil separator control 


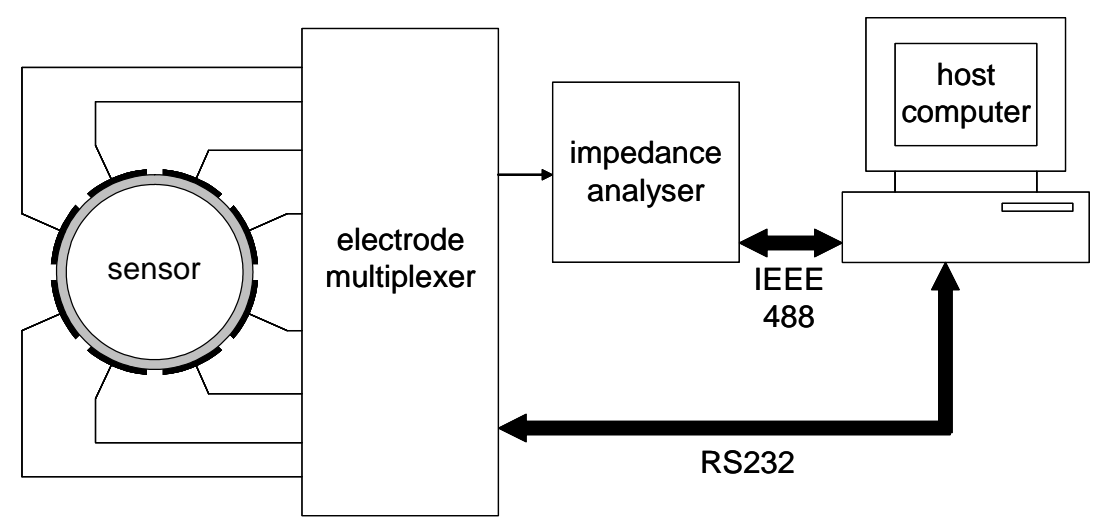

Fig. 11 Impedance analyser based ECT system

As shown in Fig. 10 (a), the ECT system is used to monitor the separation process and measure interface levels between air and oil, and oil and water by knowledge-based analysis of tomographic images (Bukhari and Yang 2004, 2005). The information is then used to control the operation of the flow rig. When the separation process finishes, the PC controls the valves to release oil to the oil storage tank and water to the water storage tank. Oil and water are pumped to the mixer and are mixed into emulsion. The mixing process is also monitored by the ECT system to determine when the mixing process should stop. The emulsion is pumped to the separator again. This test rig provides a facility for investigation of monitoring and control of oil separation processes, using the most advanced non-intrusive and noninvasive tomographic technique.

Because the impedance analyser can measure both capacitance and loss conductance, it is possible to further develop the impedance analyser based tomography system into a dual modality system, so that it can deal with saline water problems.

Although some companies, such as Tracerco, have mentioned oil separator control, we believe that the work carried out at the University of Manchester is a first in investigating oil separator control, aiming to understand the separation process and to enhance the efficiency of oil separators.

\section{Conclusions and discussion}

While many different types of sensing principles have been used for level measurement, only a few types of sensors have been used for multi-interface level measurement in crude oil separators, including $\gamma$-ray, ultrasonic, capacitance and inductance. Although each of them has its own features and woks in some extent, none of them is satisfactory for the use in industry yet. $\gamma$-ray systems have radiation problems. Ultrasonic transducers may not be able to provide accurate measurements. Capacitance sensors suffer from conductive water. Inductive sensors cannot provide information on oil and gas. In the near future, it is likely that a new type of multi-modality segmented sensor can be developed. As Hjertaker et al. suggested (2001), a combination of a capacitance sensor array and a Hall-effect sensor array may be ideal as a multi-modality sensor for multi-interface measurement.

In many other situations rather than crude oil separators, multi-interface measurement is also needed. The following are some examples (Yang 2001, Enraf-Nonius 1988). In petrol stations, a petrol/kerosene storage tank may contain petrol/kerosene on the top and water in the bottom. It is desirable to have a multi-interface level measurement system, which can locate not only the level of petrol/kerosene, but also tell if there is water layer in the bottom, because water in fuel would cause problems to engines. Accurate measurement of interface levels also helps identify leakage of the tanks, which often happens. In airport, it is surprising that how old approach is used to tell how much petrol in the storage tanks. Also, there is no efficient instrument, which can be used to tell if there is any water in the bottom of the tanks or to tell if any tank leaks.

As for the three-phase separator TORE SEP ${ }^{\circledR}$ developed by Merpro (see Fig. 2), it would be an ideal type of on-line separator if it were working as designed. Considering complexity in flow dynamics inside of the separator, it is necessary to use some advanced tool, such as tomography, to understand the separation process, to optimise the design and even to control the operation. 
Multi-interface level measurement systems are needed by the oil industry to monitor and control crude oil separators. The corresponding instrumentation will be available, either conventional type or tomographic type.

\section{Acknowledgments}

The author would like to take this opportunity to thank all his colleagues and students, who made contribution to the research into multi-interface level measurement, including Professor Maurice Beck, Dr Roger Waterfall, Dr Mike Brant, Yue Wen Chen, Dr Cheng Gang Xie, Dr Nas Chondronasios, Yahia Asran, Tariq Syed, Mohmed Akhneifer, Faisal Bukhari, Abdulgadir Hwili, Idris Ismail and Professor Tom Dyakowski of the University of Manchester (former UMIST), and Professor Hua Xiang Wang from Tianjin University.

\section{References}

1. Akhneifer A E M (1999), Development of a multi-interface level measurement system, MSc dissertation, Dept. of Electrical Engineering and Electronics, UMIST

2. Asran A (1998), Development of a multi-interface level measurement system using a segmented capacitance sensor, MSc dissertation, Dept. of Electrical Engineering and Electronics, UMIST

3. Beck M S, Waterfall R C, Williams R A, Yang W Q, Chen Y W, Banner A and Brant M R (1994), Interface measurements in three-phase separators, Final report of project PI5 "Design and instrumentation of primary separation systems, Phase I”, Process Tomography Group, UMIST

4. Brant M R, Yang W Q, Wang H X, Chen Y W, Xie C G, Hoyle B S, Lenn C P and Beck M S (1993), Interface measurement in three phase separators and pipelines, IBC Conference on developments in production separation systems, London

5. Bukhari S F A and Yang W Q (2004), Tomographic imaging technique for oil separator control, Proc. of $3^{\text {rd }}$ Int. Symp. on Process Tomography in Poland, 9-10 Sept., Lodz, Poland, pp 27-31

6. Bukhari S F A and Yang W Q (2005), New trends in multi-interface level sensors for monitoring oil separators, Proc. of $3^{\text {rd }}$ Int. Symp. on Sensor Science, 18-21 July, Juelich, Germany, pp 179-184

7. Chondronasios A, Yang W Q and Nguyen V T (2001), Impedance-analyser-based tomography system, Proc. of $2^{\text {nd }}$ World Congress on Industrial Process Tomography, 29-31 Aug., Hannover, Germany, pp 573-579

8. Christian Michelsen Research AS (2005), Ultrasonic interface level detector, http://www.cmr.no/ avd10/nedlasting/Brosjyre_UID.pdf

9. Darwood M, James K, Jackson P and Hewitt P (2003), Density profiling in multiphase systems using Gamma ray absorption, Proc. of $3^{\text {rd }}$ World Congress on Industrial Process Tomography, 2-5 Sept., Banff, Canada, pp 870-875

10. Dyakowski T, Hale J M, Jaworski A, White N M, Nowakowski A, Meng G and Rwifa S (2005), Dual-modality probe for characterization of heterogeneous mixtures, IEEE Sensors Journal, 5 (2), pp 134-138

11. Endress+Hauser (2005), Rod probes 11500 Z, 11500 ZM, http://ww.endress.com/eh/products/en/ home.nsf/contentview

12. Enraf-Nonius BV (1988), Level gauging system for service stations user manual

13. Hjertaker B T, Johansen G A and Jackson P (2001), Recent developments in hydrocarbon separator interface imaging, Proc. of SPIE, 4188, pp 81-92

14. Holstad M B, Johansen G A, Jackson P and Eidsnes K S (2003), Scattered Gammar radiation utilized for level measurements in gravitational separators, Proc. of $3^{\text {rd }}$ World Congress on Industrial Process Tomography, 2-5 Sept., Banff, Canada, pp 181-186

15. Isaksen $\varnothing$ and Nordtvedt J E (1992), A new reconstruction algorithm for process tomography, Meas. Sci. Technol., 4, pp 1464-1475

16. Isaksen Ø, Dico A S and Hammer E A (1994), A capacitance based tomography system for interface measurement in separation vessels, Meas. Sci. Technol., 5, pp 1262-1271

17. Jaworski A J, Dyakowski T and Davies G A (1999a), A portable capacitance probe for detection of interface levels in multi-phase flows - A case study, Proc. of $1^{\text {st }}$ World Congress on Industrial Process Tomography, 14-17 April, Buxton, UK, pp 289-296

18. Jaworski A J, Dyakowski T and Davies G A (1999b), A capacitance probe for interface detection in oil and gas extraction plant, Meas. Sci. Technol., 10, pp L15-L20 
19. Jaworski A J and Dyakowski T (2005), Measurements of oil-water separation dynamics in primary separation systems using distributed capacitance sensors, Flow Measurement and Instrumentation, 16 (2-3), pp 113-127

20. Lees R P (2005), Increase oil production and reduce chemical usage through separator level measurement by density profiling, http://www.isa.org/journals/intech/ETCON2046.pdf

21. Meng G, Jaworski A J, Dyakowski T, Hale J M and White N M (2005), Design and testing of a thick-film dual-modality sensor for composition measurements in heterogeneous mixtures, Meas. Sci. Technol.,16 (4), pp 942-954

22. Merpro (2005), Separation TORE SEP and AZGAZ®, http://www.merpro.com/PRNFrontend/ upload/tplt main1a .asp?page $=2100002151$

23. Schüller R B, Solbakken T, Engebretsen B and Halleraker M (2001), Water concentrations and interface positions by single electrode capacitance probes, Proc. of $2^{\text {nd }}$ World Congress on Industrial Process Tomography, 29-31 Aug., Hannover, Germany, pp 644-651

24. Shell International Research (1984), Level Gauge, European Patent Application No. 84201889.7

25. Shi T M, Xie C G, Huang S M, Williams R A and Beck M S (1991), Capacitance-based instrumentation for multi-interface level measurement, Meas. Sci. Technol., 2, pp 923-933

26. Syed T (2004), 2D/3D magnetic arrays for imaging, MSc dissertation, Dept. of Electrical Engineering and Electronics, UMIST

27. Tracerco (2005), Specialist measurement instruments - Tracerco Profiler, http://www.tracerco.com/ products-specialistinstruments-profiler.htm

28. Wang H X, Yin W L, Yang W Q and Beck M S (1996), Optimum design of segmented capacitance sensing array for multi-phase interface measurement, Meas. Sci. Technol., 7, pp 79-86

29. Wang H X and Yin W L (1997), A study of the detection mechanism and the reconstruction algorithm for multi-phase interface level measurement, Meas. Sci. Technol., 8, pp 1289-1294

30. Yang W Q, Brant M R and Beck M S (1994), A multi-interface level measurement system using a segmented capacitance transducer for oil separators, Meas. Sci. Technol., 5 (9), pp 1177-1180

31. Yang W Q (2001), Multi-interface liquid level testing system, Chinese Patent, CN2443351U 